\title{
Phenytoin in Topical Formulations Augments Pain Reduction of Other Analgesics in the Treatment of Neuropathic Pain
}

\author{
David J Kopsky* and Jan M Keppel Hesselink \\ Institute for Neuropathic Pain, Amsterdam \& Bosch en Duin, The Netherlands
}

*Corresponding author: David J Kopsky, MD, Institute for Neuropathic Pain, Vespuccistraat 64-III, 1056 SN, Amsterdam, The Netherlands, Tel: +31-6-28671847, E-mail: info@neuropathie.nu

\begin{abstract}
Topical analgesic formulations are gaining interest for the treatment of peripheral neuropathic pain since the beginning of 2000 . Advantages of topical analgesics over oral medication are the absence of systemic side effects and drug-drug interactions, higher concentrations of active compound at the pain area, fast onset on action, improvement of compliance, and no risk of abuse. In many peripheral neuropathic pain states the pain area is small and thus topical analgesics are suitable. Most patients experience pain reducing effect within 30 minutes after application of compounded topical analgesics, such as creams containing amitriptyline, ketamine, baclofen, or clonidine. This helps to quickly identify responders on selected analgesic creams.

Unfortunately, in some patients the duration of topical applied analgesic formulations is short and/or the reduction of pain is insufficient. Therefore, strategies to prolong the duration and intensify the analgesic effect are needed. We discovered that phenytoin augments the effects of topical analgesics, leading to a faster onset of action, a longer duration of analgesia, and an intensified pain relieving effect. We will present 6 cases in which phenytoin $5 \%$ or $10 \%$ lead to enhanced therapeutic effects of topical applied amitriptyline $10 \%$, ketamine $10 \%$, baclofen $5 \%$, or clonidine $0.2 \%$ creams in the treatment of neuropathic pain. We also present a fast topical analgesic response-test to identify responders within a 30 minutes' assessment period after a test application. Responders are defined as experiencing at least 2 points reduction of pain intensity, as measured with the 11-point numerical rating scale. Responders subsequently are treated with the analgesic cream as tested.
\end{abstract}

\section{Introduction}

Since 2000 topical analgesics have been gaining interest for the treatment of peripheral neuropathic pain. This is most probably due to the fact that topical analge- sics have a number of advantages over oral medication, such as the absence of systemic side effects and drugdrug interactions, fast onset of pain relief, high concentration of the active compound at the application area, improvement of compliance, and no risk of abuse [1]. These advantages are based on the fact that topical analgesics have a peripheral mechanism of action, without the need to enter the bloodstream. Especially in geriatric patients with neuropathic pain, with high risk of undesirable side effects, drug-drug interactions and/or altered metabolization due to intake of multiple medications, topical analgesics should be considered as a first line treatment option [2].

In our Institute for Neuropathic Pain we developed and tested topical compounded analgesics, such as ketamine $10 \%$, amitriptyline $10 \%$, and baclofen $5 \%$ creams [3-10]. Most responders experience pain reducing effect within 30 minutes after application. Using a topical analgesic response-test, responders can be identified within a 30 minutes assessment period after a test application. A responder is defined as least 2 points reduction of pain intensity, as measured with the 11-point numerical rating scale (NRS). After identifying the responder as such, we prescribe the topical analgesic which was tested.

Unfortunately, some of our patients experience pain relief only for a short period of time (e.g. 1 to 2 hours). In our search to solve this problem we discovered that the old anticonvulsant phenytoin has remarkable properties. Phenytoin added to other active compounds in topical formulations leads to augmentation of effects, such as a faster onset of action, a longer duration of 
analgesia, and/or an intensified pain relieving effect. Topical phenytoin is currently only known to accelerate healing of chronic ulcers and wounds $[11,12]$. In the present study 6 cases in which phenytoin is added as a booster to an analgesic in a topical formulation in patients suffering from a variety of neuropathic pain syndromes are presented.

\section{Case Presentations}

\section{Case 1: Small fiber neuropathy (SFN)}

A 57-year-old man suffered from symptoms of SFN, being treated with pregabalin, without sufficient pain reduction. The patient complained of burning pain, tingling and coldness in feet and hands, aggravating while standing and walking. One part of the neuropathic pain area (situated at the most painful foot) was treated with ketamine $10 \%$ cream. This resulted after 10 minutes in a slight reduction of burning pain, although the tingling aggravated. Another not yet treated part of the most painful foot was subsequently treated with phenytoin $10 \%$ cream. This resulted within a timeframe of 10 minutes in a reduction of burning pain as well as of coldness. Adding phenytoin cream on top of the ketamine cream resulted within 5 minutes in a further pain reduction from 6.5 to 3 on the NRS. Surprisingly, the aggravated tingling provoked by ketamine cream disappeared totally after the application of the phenytoin cream. The overall finding was expressed by the patient as: "it all feels now peaceful and quit in the area where the two creams were applied". The improvement lasted for 24 hours.

\section{Case 2: Diabetic neuropathic pain}

A 69-year-old man suffered since 2007 from diabetic neuropathic pain in both forefeet and scored the pain as 9 on the NRS. The pain was characterized by burning, electric shocks, tingling, pins and needles, allodynia (pain in response to normally non-painful stimuli) after soft stroking. Especially the allodynia in his left foot was bothering him during night. Pregabalin $75 \mathrm{mg}$ twice daily did not have any effect. Administering ketamine $10 \%$ cream [8], resulted in pain reduction from 9 to 5.5 on the NRS, with an onset of effect of 25 minutes and duration of pain reduction of 6.5 hours. Adding phenytoin $10 \%$ on top of ketamine $10 \%$ cream resulted in an onset of effect of around 5 minutes, reduction of pain to 2.5 on the NRS, and a prolonged duration of effect of 11 hours.

\section{Case 3: Chronic idiopathic axonal polyneuropathy (CIAP)}

A 63-year-old man suffered since 2012 of CIAP with pain in both feet ( 8 on the NRS) with the following characteristics: burning, pins and needles and numbness. Oral amitriptyline did not have any pain relieving effect. Topical clonidine $0.2 \%$ cream reduced symptoms after 15 minutes: the sensation of pins and needles is reducd to 5 and the burning pain to 2.5 on the NRS. The duration of the effect was 6 hours only, resulting in disrupt- ed sleep. After adding phenytoin $5 \%$ to clonidine $0.2 \%$ cream, the onset of effect was within 5 minutes, the sensation of pins and needles was more reduced to a score of 2.5, and burning pain to a score of 0 on the NRS. The duration of the effect was 10 hours; thus, he could sleep the whole night through. He also could reduce the application of cream from 3 to 2 times daily after phenytoin was added.

\section{Case 4: Chemotherapy induced Polyneuropathy (CIPN)}

A 67-year-old man suffered since 2015 of CIPN a developed during the treatment with oxaliplatin of a metastasized rectal carcinoma. He experienced his pain as tingling, pins and needles and numbness in both feet and complained of coldness of the feet. He scored his complaints as 5 on the NRS. The combination cream of lidocaine 3\% together with isosorbide dinitrate $0.4 \%$ cream reduced his pain to a 4 on the NRS. To check the difference in effect between phenytoin $10 \%$ cream, compared to the combination of phenytoin $10 \%$ and baclofen $5 \%$ cream, phenytoin $10 \%$ cream was applied on the left foot, and the combination cream was applied on the right foot. He experienced pain reduction of the left foot from 4 to 3 and the right foot from 4 to 1 on the NRS. The booster phenytoin $10 \%$ cream applied alone had only a slight symptom reducing effect, while in combination with baclofen $5 \%$ cream a clear symptom reduction was seen.

\section{Case 5: CIPN}

A 48-year-old man suffering from acute leukemia was treated with mitoxantrone and etoposide in July 2014. The chemotherapy caused hand-foot syndrome (redness and edema), and neuropathic pain in the feet. He described the pain as burning, tingling, pins and needles, and scored this pain with an 8.5 on the NRS in November 2015. Physical examination revealed hypoesthesia for pinprick and touch, and allodynia. Amitriptyline 10\% cream reduced within 8 minutes the pain to a score of 0 on the NRS, though only for 1 to 1.5 hours. In October 2016, he scored the neuropathic pain as 6 on the NRS. He was subsequently treated with phenytoin $5 \%$ cream, which also resulted in complete disappearance of the neuropathic pain, though the duration was clearly longer: 3.5 hours, with an onset of effect of 15 minutes after application. The combination of phenytoin $5 \%$ and amitriptyline $10 \%$ resulted in complete disappearance of the pain, with a prolonged effect of in total 8 hours and an onset of action of 3 minutes. He could sleep again the whole night without being disturbed.

\section{Case 6: Neuropathic pain after Guillain-Barre syn- drome}

A 63-year-old man suffered from Guillain-Barre syndrome in 1980 . He recovered with minor sequelae: numbness of the feet, reduced facial expressions, and diminished motor function of the left hand. In 2011 he experienced stabbing pain in his big left toe ( 8.5 on the 
NRS) especially at night. Oral pregabalin had some pain reducing effect, but he experienced bothersome side effects: anxiety and depression. Baclofen $5 \%$ cream clearly reduced the pain completely, but had to be applied 2 to 4 times during the night. Adding phenytoin $5 \%$ to baclofen $5 \%$ cream prolonged the pain reducing effect considerably and only one application before sleeping was required.

None of the above described patients reported any local or systemic side effects, the creams were well tolerated and administration was conducted without problems.

\section{Discussion}

Recently, we reported that phenytoin could augment the effects of ketamine $10 \%$ and baclofen $5 \%$ in trigeminal neuralgia [13]. Adding phenytoin to analgesics or co-analgesics in a topical formulation, led to 1 ) faster onset of action, 2) longer duration of analgesia, and 3) more pronounced analgesia in neuropathic pain. The mechanisms of action of phenytoin are pleiotropic, related to a number of ion channels and other targets, and thus synergistic effects when combined with the analgesics described below are to be expected. Phenytoin is well-known as a non-selective voltage-gated sodium channel $\left(\mathrm{Na}_{\mathrm{v}}\right)$ stabilizer [14]. Furthermore, phenytoin inhibits voltage dependent L-type calcium channels [15], and influences the GABA receptor [16]. However, the full spectrum of phenytoin's mechanism of action is still unfolding.

An intact nociceptor function in the epidermis is essential to achieve analgesia with topical analgesics. Campell, et al. examined this phenomenon in a randomized controlled trial in patients suffering from painful diabetic neuropathy [17]. The effectiveness of topical clonidine $0.1 \%$ in reducing pain is dependent on the severity or intensity of pain measured 30 minutes after capsaicin $0.1 \%$ application.

\section{Faster onset of action}

The faster onset of action after adding phenytoin to an analgesic cream, is most probably due to a synergistic effect of phenytoin and the other active compound. As described in case 2, amitriptyline and ketamine applied separately led to an onset of action within 10 to $15 \mathrm{mi}-$ nutes, while combining either amitriptyline or ketamine with phenytoin led to an onset of action of less than 5 minutes. A fast onset of effect of topical analgesics in literature, is only sparsely described. To our knowledge, only one publication showed that a combination of compounds showed a faster onset of action than the single compounds. In a patent from 1997 a faster onset of action was described after topical application of the combination ketamine and amitriptyline, compared to the topical single compounds [18]. A topical analgesic has to penetrate the lipophilic stratum corneum, which is around $0.02 \mathrm{~mm}$ thick [19], in order to reach the nociceptive nerve endings located in the stratum spinosum. The lipophilic nature of phenytoin, the small size of the molecule (252 Dalton), the selected high concentration $(10 \%)$, and the presence of penetration enhancers in the topical formulation might explain the fast onset of effect.

The fast onset of action by adding phenytoin to another analgesic in a topical formulation, could also reside in the fact that phenytoin might influence epidermal keratinocytes, which on their turn can influence the nociceptors reaching up to the upper layers of the stratum spinosum [20]. $\mathrm{Na}_{v}$ on epidermal keratinocytes in pain syndromes are upregulated [21], and activation results in ATP release leading to activation of $P 2 X$ receptors on nociceptive sensory endings. Phenytoin as a $\mathrm{Na}_{v}$ stabilizer can thus stabilize the upregulated $\mathrm{Na}_{\mathrm{v}}$ of keratinocytes in pain syndromes $[14,22]$.

The fast onset of action further suggests a role of peripheral sensitization in these pain syndromes. Although central sensitization can be influenced with topical analgesics [23], in that case one might expect the onset of effect to be longer than described in our cases.

\section{Longer duration of analgesia}

The mechanism of the prolonged pain reduction when adding phenytoin to a topical formulation with ketamine, baclofen or clonidine is not known. From other compounds used as adjuvantia it is known that these prolonged effects of local anesthetics. For example, prolongation of pain reducing effect is observed when using magnesium sulfate as an adjuvant to local anesthetics in perineural nerve blocks [24]; in neuraxial blocks the adjuvant dexmedetomidine to local anesthetics prolongs the duration of analgesia [25].

\section{More pronounced analgesia}

The use of multiple oral analgesics from different classes gives a more pronounced pain reduction compared to single oral analgesics [26-29]. Also there is some evidence that combinations of topical (co)analgesics lead to a more pronounced pain reduction compared to topical monotherapy [30-32]. Influencing multiple receptors and/or enhancing affinities of the main analgesic compounds might be the explanation for pronounced pain reduction.

In conclusion, phenytoin seems to augment other analgesic compounds when added to topical formulations. Its broad receptor and ion channel affinity might explain the synergistic effects we found when combining phenytoin with analgesic compounds. Our patients have not reported any side effects in the treatment of neuropathic pain, when treated with the combination of topical phenytoin and other analgesic compounds. Topical analgesics have several advantages over oral analgesics, such as the absence of systemic side effects, 
low propensity for drug-drug interactions, while leading to higher concentrations of active compound at the pain area, a fast onset of pain relief, improvement of compliance, and no risk of abuse. We recommend to perform a response-test before prescribing a topical analgesic, in order to optimize the treatment.

\section{Conflict of Interest}

The authors are holders of two patents: 1) Topical phenytoin for use in the treatment of peripheral neuropathic pain and 2) Topical pharmaceutical composition containing phenytoin and a (co-)analgesic for the treatment of chronic pain. The authors report no other conflicts of interest in this work.

\section{References}

1. Anitescu M, Benzon HT, Argoff CE (2013) Advances in topical analgesics. Curr Opin Anaesthesiol 26: 555-561.

2. Kopsky DJ (2016) Topical analgesia is a viable option. J Pain Relief 5: 245.

3. Kopsky DJ, Keppel Hesselink JM (2012) High doses of topical amitriptyline in neuropathic pain: two cases and literature review. Pain Pract 12: 148-153.

4. Kopsky DJ, Keppel Hesselink JM (2010) A new combination cream for the treatment of severe neuropathic pain. $J$ Pain Symptom Manage 39: e9-e10.

5. Kopsky DJ, Keppel Hesselink JM (2013) Neuropathic pain as a result of acromegaly, treated with topical baclofen cream. J Pain Symptom Manage 46: e4-e5.

6. Kopsky DJ, Keppel Hesselink JM, Bhaskar A, Hariton G, Romanenko V, et al. (2015) Analgesic effects of topical ketamine. Minerva Anestesiol 81: 440-449.

7. Kopsky DJ, Liebregts R, Keppel Hesselink JM (2012) Central neuropathic pain in a patient with multiple sclerosis treated successfully with topical amitriptyline. Case Rep Med 2012: 471835.

8. Keppel Hesselink JM, Kopsky DJ (2013) Treatment of chronic regional pain syndrome type 1 with palmitoylethanolamide and topical ketamine cream: modulation of nonneuronal cells. J Pain Res 6: 239-245.

9. Keppel Hesselink JM, Kopsky DJ (2016) Topical analgesic creams and nociception in diabetic neuropathy: towards a rationale fundament. Clin Case Rep Rev 2: 500-502.

10. Keppel Hesselink JM, Kopsky DJ, Sajben NL (2014) Vulvodynia and proctodynia treated with topical baclofen $5 \%$ and palmitoylethanolamide. Arch Gynecol Obstet 290: 389-393.

11. Shorvon SD (2009) Drug treatment of epilepsy in the century of the ILAE: the first 50 years, 1909-1958. Epilepsia 50: 69-92.

12. Bhatia A, Prakash S (2004) Topical phenytoin for wound healing. Dermatol Online J 10: 5.

13. Kopsky DJ, Keppel Hesselink JM (2017) Phenytoin in topical formulations augments pain reduction of other topically applied analgesics in the treatment of trigeminal neuralgia. J Clin Anesth 38: 154-155.

14. Clare JJ (2010) Targeting voltage-gated sodium channels for pain therapy. Expert Opin Investig Drugs 19: 45-62.

15. Patejdl R, Leroux AC, Noack T (2015) Phenytoin inhibits contractions of rat gastrointestinal and portal vein smooth muscle by inhibiting calcium entry. Neurogastroenterol Motil 27: $1453-1465$.

16. Cunningham MO, Dhillon A, Wood SJ, Jones RS (2000)
Reciprocal modulation of glutamate and GABA release may underlie the anticonvulsant effect of phenytoin. Neuroscience 95: 343-351.

17. Campbell CM, Kipnes MS, Stouch BC, Brady KL, Kelly M, et al. (2012) Randomized control trial of topical clonidine for treatment of painful diabetic neuropathy. Pain 153: 1815-1823.

18. Frome BM (1995) Inventor. The preparation of topical regional compositions for the relief of pain 1995.

19. Bohling A, Bielfeldt S, Himmelmann A, Keskin M, Wilhelm KP (2014) Comparison of the stratum corneum thickness measured in vivo with confocal Raman spectroscopy and confocal reflectance microscopy. Skin Res Technol 20: 50-57.

20. Keppel Hesselink JM, Kopsky DJ, Bhaskar AK (2017) Skin matters! The role of keratinocytes in nociception: a rational argument for the development of topical analgesics. J Pain Res 10: 1-8.

21. Zhao P, Barr TP, Hou Q, Dib-Hajj SD, Black JA, et al. (2008) Voltage-gated sodium channel expression in rat and human epidermal keratinocytes: evidence for a role in pain. Pain 139: 90-105.

22. Qiao X, Sun G, Clare JJ, Werkman TR, Wadman WJ (2014) Properties of human brain sodium channel alpha-subunits expressed in HEK293 cells and their modulation by carbamazepine, phenytoin and lamotrigine. $\mathrm{Br} \mathrm{J}$ Pharmacol 171: 1054-1067.

23. Baron R, Hans G, Dickenson AH (2013) Peripheral input and its importance for central sensitization. Ann Neurol 74: 630-636.

24. Li M, Jin S, Zhao X, Xu Z, Ni X, et al. (2016) Does Magnesium Sulfate as an Adjuvant of Local Anesthetics Facilitate Better Effect of Perineural Nerve Blocks? A Meta-analysis of Randomized Controlled Trials. Clin J Pain 32: 1053-1061.

25. Wu HH, Wang HT, Jin JJ, Cui GB, Zhou KC, et al. (2014) Does dexmedetomidine as a neuraxial adjuvant facilitate better anesthesia and analgesia? A systematic review and meta-analysis. PLoS One 9: e93114.

26. Gilron I, Chaparro LE, Tu D, Holden RR, Milev R, et al. (2016) Combination of pregabalin with duloxetine for fibromyalgia: a randomized controlled trial. Pain 157: 1532-1540.

27. Gilron I, Tu D, Holden RR, Jackson AC, DuMerton-Shore D (2015) Combination of morphine with nortriptyline for neuropathic pain. Pain 156: 1440-1448.

28. Gilron I, Bailey JM, Tu D, Holden RR, Jackson AC, et al. (2009) Nortriptyline and gabapentin, alone and in combination for neuropathic pain: a double-blind, randomised controlled crossover trial. Lancet 374: 1252-1261.

29. Gilron I, Bailey JM, Tu D, Holden RR, Weaver DF, et al. (2005) Morphine, gabapentin, or their combination for neuropathic pain. N Engl J Med 352: 1324-1334.

30. Costantino C, Kwarecki J, Samokhin AV, Mautone G, Rovati $S$ (2011) Diclofenac epolamine plus heparin plaster versus diclofenac epolamine plaster in mild to moderate ankle sprain: a randomized, double-blind, parallel-group, placebo-controlled, multicentre, phase III trial. Clin Drug Investig 31: 15-26.

31. Myrer JW, Johnson AW, Mitchell UH, Measom GJ, Fellingham GW (2011) Topical analgesic added to paraffin enhances paraffin bath treatment of individuals with hand osteoarthritis. Disabil Rehabil 33: 467-474.

32. Somberg JC, Molnar J (2015) Retrospective Evaluation on the Analgesic Activities of 2 Compounded Topical Creams and Voltaren Gel in Chronic Noncancer Pain. Am J Ther 22: $342-349$ 